\title{
Agro-Industrial Waste-Mediated Green Synthesis of Silver Nanoparticles and Evaluation of Its Antibacterial Activity
}

\author{
Rahul Shankar Tade ${ }^{1}$, Sopan Namdev Nangare ${ }^{1}$, Pravin Onkar Patil ${ }^{2}$ \\ ${ }^{1}$ H. R. Patel Institute of Pharmaceutical Education and Research, Shirpur, Dist. Dhule Maharashtra, India-425405. \\ ${ }^{2}$ Department of Chemistry, H. R. Patel Institute of Pharmaceutical Education and Research Shirpur, Dist. Dhule, Maharashtra, \\ India-425405. \\ Corresponding author. E-mail: taderahul2011@gmail.com; Tel.: +91 7798806826
}

Received: Oct. 9, 2019; Accepted: Feb. 21, 2020; Published: Feb. 21, 2020.

Citation: Rahul Shankar Tade, Sopan Namdev Nangare, and Pravin Onkar Patil, Agro-Industrial Waste-Mediated Green Synthesis of Silver Nanoparticles and Evaluation of Its Antibacterial Activity. Nano Biomed. Eng., 2020, 12(I): 57-66.

DOI: $10.5101 /$ nbe.v12i1.p57-66.

\begin{abstract}
The development of immaculate etiquette for the green and rapid synthesis of Ag NPs with a natural reducing agent is the spearhead of the expanding field of nanotechnology. Different scientific fraternity with novel natural reducing agents has been contributing numerous strategies daily. Though there is a submerging of many natural reducing agents, still there are plenty of natural precursors remained to be explored. In this research, we fruitfully attempted the synthesis of silver nanoparticles using agrofood industrial waste Tamarindus indica shell-husk extract (TSE) as a natural reducing agent. The prepared silver nanoparticles and their stability in different $\mathrm{pH}$ were investigated using ultravioletvisible spectroscopic analysis. Morphological characters were examined using scanning electron microscope (SEM) and transmission electron microscopy (TEM) analysis. The structural and elemental compositions were depicted by Fourier-transform infrared spectroscopy (FTIR) and energy-dispersive $\mathrm{X}$-ray (EDX) analysis, respectively. Moreover, we emphasized on the molecular mechanism involving in the TSE mediated synthesis of Ag NPs. The inherent antimicrobial activity was investigated using agar plate method against both gram-positive and gram-negative species with gentamycin as a control standard for comparison.
\end{abstract}

Keywords: Green synthesis of Ag NPs; Tamarinds shell-husk extract; Effect of pH; Onepot synthesis; Antimicrobial activity

\section{Introduction}

In the recent era, nanobiotechnology has benediction the advantages of the synthesis of nanostructures using living organisms such as plant and microbes. Plant-mediated synthesis of nanoparticles could be advantageous over additional environmentally benevolent biological processes as it eliminates the process involving toxic chemicals and reactants. Biosynthetic processes for nanoparticles would be more expedient if nanoparticles are produced extracellularly using plants or their extracts and in a controlled manner according to their size, dispersity, and shape [1]. Plant-mediated biological synthesis of nanoparticles is gaining significance due to its ease and eco-friendliness. Biosynthetic processes would be more useful if the silver nanoparticles (Ag NPs) were produced using plants or their extracts in a controlled approach according to their dispersity, shape, and size [2]. Although it is a well-known fact 
that the silver metal exerts antimicrobial activity and antioxidant activity, this natural gift will be adopted using a simple green synthesis of Ag NPs using plants with greener approach [3, 4]. Currently most of the areas focusing on the application of Ag NPs in a broad area such as anticancer, antibacterial, larvicidal activity and antifungal agents, in textile engineering, waste water treatment, and silver-based consumer products [5]. A new concept of the morphological dependence of the antimicrobial activity of the Ag NPs was added by Cheon et al. [7]. Since the difference in the $\mathrm{Ag}^{+}$ion release depended on the shape, it would be possible to control the antimicrobial activity by controlling the shape and size of the Ag NPs [6]. Effect of physicochemical conditions on biosynthesis and antimicrobial efficiency of Ag NPs by a marinederived fungus was first reported by Shankar et al. [9]. They attempted the synthesis of silver nanoparticles by a marine fungus Aspergillus, and successfully obtained narrowly dispersed Ag NPs by altering the reaction condition. Moreover, the antibacterial activity of these biosynthesized Ag NPs was tested against two gram-negative and two gram-positive bacterial strains. The Ag NPs synthesized at $\mathrm{pH} 10$ were found to be the most effective against test bacteria, from which it can be concluded that $\mathrm{pH}$ plays an important role [7]. Plants or their extracts have also been explored in the synthesis of Ag NPs alone using silver ions as substrate. Aqueous silver nitrate solution, after reacting with leaf extract, led to the rapid formation of crystalline silver nanoparticles, which assembled in the reaction medium into quasi-linear superstructures [8]. The use of plant biomasses or plant extracts is comparatively simpler and more cost-effective [9]. This is especially useful if low-value agricultural waste can be used for this rationale which complies with the notion of "waste to wealth". Plant-mediated synthesis of metallic nanoparticles has been shown to produce nanoparticles with shapes and sizes that are comparable with those produced through chemical and physical methods [10]. Plants are known to harbor a big range of metabolites that are most likely to be responsible in the green synthesis of metal nanoparticles since anyone can apply the different biotechnological approach for the same $[11,12]$.

Tamarindus indica L., usually known as the tamarind tree, is well known for its various applications. The most commonly used part is the fruit in food additives and related cuisine. The pulp constitutes about $35-50 \%$ in the ripe fruit, the shell and fiber account for $11-30 \%$, and the seed about $25-40 \%$. Tamarind is mostly used as a health remedy throughout Asia, Africa, and the Americas. Other Tamarind products, leaves, fruits and seeds have been extensively used in Indian Ayurvedic medicine and traditional African medicine [13, 14]. Instead of the valuable use of pulp, the most valuable part is the outer hull of the tamarind legume have been explored for the different types of water remediation applications [15]. The influence of shaking and stirring speed on the defluoridation behavior of tamarind fruit shell derived activated carbon was studied by Sivsankar et al., The fluoride removal efficacy of the prepared material as a function of $\mathrm{pH}$, initial fluoride concentration, sorbent dose, and co-ionic interference was investigated. They utilized kinetic and isotherm models were used to interpret the nature of the fluoride sorption onto the presence of $\mathrm{CaCO}_{3}$ compounds in the carbon matrix which is apparent from the results of defluoridation studies [16]. Although there is plenty of literature revealed the used of natural reducing agents such as leaves extract of neem [17], tea [18], eucalyptus[19], rose [20], Aloe vera [21] etc. However, earlier researches used fresh leaves of plants and tedious processing parameters. We claimed the truly green approach without harming natural flora. Moreover, we firstly explored a green approach for the one-step synthesis of Ag NPs using Tamarindus indica shell-husk (TIS) extract as the reducing agent and stabilizer. We also focused on we Moreover, we investigated the physiochemical properties of the TIS extract and Ag NPs with respect to the different processing parameters. The antimicrobial properties of the synthesized Ag NPs against E. coli, Streptococcus aureus, and Staphylococcus aureus were also evaluated.

\section{Experimental}

\section{Materials and procedure for the preparation of extracts}

Silver nitrate $\mathrm{AgNO}_{3}$ was purchased from SigmaAldrich Chemicals and used as received. Hydrochloric acid and sodium hydroxide were purchased from Loba-Chemie and used as received. Deionized water was used throughout the reactions. All glassware was washed with dilute nitric acid and distilled water. A stock solution of $\mathrm{AgNO}_{3}$ of different molarities was prepared by dissolving in deionized water. Tamarind shells were collected from the Jain Food Park, Jam Production Industry Jalgaon, Maharashtra. 


\section{Preparation of Tamarind shell-husk extract}

The method of preparation of Tamarind shellhusk extract (TSE) of the plant was used as given in the literature [22] with slight modification. In brief, collected tamarind shell (separated from fruit pulp) washed thoroughly with tap water followed by double distilled water to remove dust and dirt and kept for drying at $60{ }^{\circ} \mathrm{C}$ for $30 \mathrm{~min}$. Then it was crushed into small pieces, weighed and ground in a mixer-grinder (Usha MG $2853500 \mathrm{~W}$ ) to convert into powder called husk. The $5 \mathrm{~g}$ of husk was then transferred into a 200 $\mathrm{mL}$ glass beaker containing $100 \mathrm{~mL}$ deionized water and boiled at $100{ }^{\circ} \mathrm{C}$ for about $30 \mathrm{~min}$. The aqueous extract was kept undisturbed for 2-3 $\mathrm{h}$ for cooling and filtered through a membrane filter $(0.45 \mu \mathrm{m})$ to remove particulate matter. The clear extract obtained was stored at $8{ }^{\circ} \mathrm{C}$ for further use.

\section{Synthesis of Ag NPs}

The overall scheme of Ag NPs synthesis is represented in Fig. 1(a). Subsequently, Ag NPs were synthesized by adding a selected volume of extract $(1.8 \mathrm{~mL})$ with a silver nitrate solution $(1 \mathrm{mM})$. The mixtures were incubated at $85-95{ }^{\circ} \mathrm{C}$ for $30 \mathrm{~min}$. and allowed to cool down and monitored periodically to

(a)

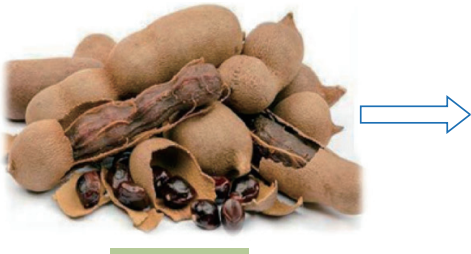

Extract

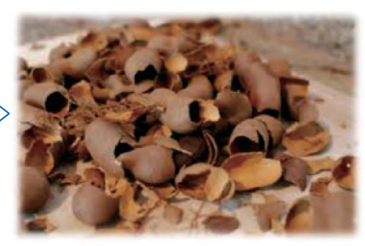

Shell Husk

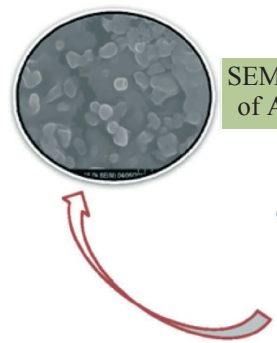

\section{SEM image} of AgNPs
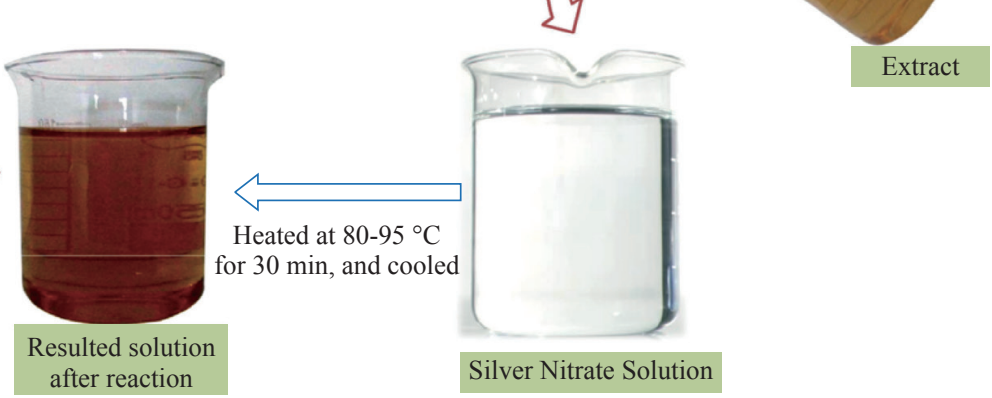

Washed with DI water and Grounded in Mixer-grinder

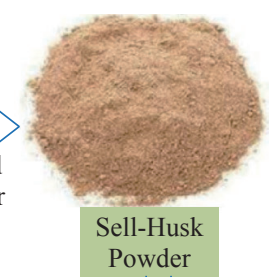

Powder

Boiled at $100^{\circ} \mathrm{C}$ for $30 \mathrm{~min}$ and cooled

(b)

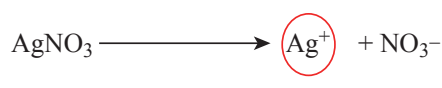<smiles>[R]c1ccc(O)c(O)c1</smiles>

General formula for TIS polyphenols

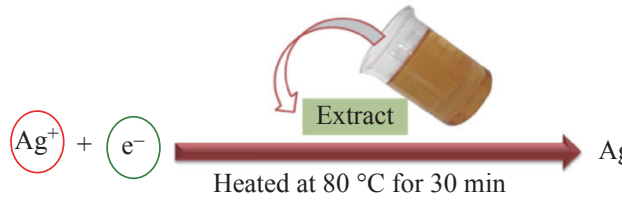

Heated at $80{ }^{\circ} \mathrm{C}$ for $30 \mathrm{~min}$

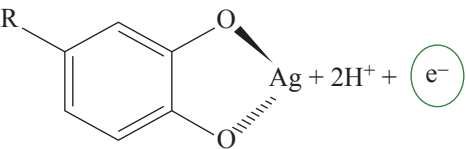

Plausible Structure of Ag@Polyphenol Complex

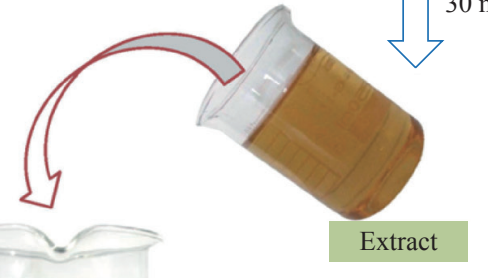

mechanism involving Ag NPs synthesis. 
evaluate the bioreduction of $\mathrm{Ag}^{+}$in aqueous solution. The same methodology was used for the previously prepared solutions of different $\mathrm{pH}$, i.e. 6.5, 7.5, 8.5, and 9.5 , respectively. The $\mathrm{pH}$ was maintained at the time of reaction; both silver nitrate solution and TSE were mixed using suitable acids (hydrochloric acid) and alkali (sodium hydroxide) solution. The change in color from colorless to dark brown indicated completion of the reaction [23], while the extract itself had light yellowish shade. After the complete reduction of $\mathrm{Ag}^{+}$ions by the TSE extract, $50 \mathrm{~mL}$ solution of $\mathrm{Ag}$ NPs was centrifuged at $6000 \mathrm{rpm}$ for $15 \mathrm{~min}$ and the resulting suspension was re-dispersed into $25 \mathrm{~mL}$ of deionized water. The same procedure was repeated three times to render nanoparticles free from additional bioorganic compounds that might be present in the solution. The purified suspension was dried using lyophilizer and kept in desiccators to prevent moist contact and simultaneously used for further analysis.

\section{Preparation of bacterial inoculum}

A 24-h nutrient broth culture of tested bacteria was grown in an orbital shaking incubator, centrifuged, washed twice with phosphate buffer, and then standardized to approximately $10^{6} \mathrm{CFU} / \mathrm{mL}$ using broth medium.

\section{Characterization of Ag NPs}

The bioreduction of $\mathrm{Ag}^{+}$by TSE in aqueous solution was monitored by measuring the spectrum of the solution within the range of 350-600 nm using UV-Visspectrophotometer (Shimadzu 1800). To investigate the stability of nanoparticles colloidal solution, the (solution with different $\mathrm{pH}$ 6.5, 7.5, 8.5, and 9.5) were kept at room temperature for one week. During this period the UV-visible spectrum of the solution was measured after one week. The size and morphology of the nanoparticles were examined using SEM-EDX (Bruker, 1530-2 FE-SEM/EDX, Japan) transmission electron microscope (Philips, CM200, 20-200 Kv at $2.4 \mathrm{~A}^{0}$ ). The sample was prepared by placing a drop of Ag-NPs on a carbon-coated copper grid and was then left to dry in the air, before transferring it to the microscope. Particle size and Zeta potential analysis were carried by using (Nanoplus-3, particulate system - Micromeritics, USA). The functional groups that might be appeared on the Ag NPs were interpreted using Fourier Transform Infrared (FTIR) spectroscopy (Shimadzu IR Affinity). All measurements were carried out in the range of $500-4500 \mathrm{~cm}^{-1}$ at a resolution of 4 $\mathrm{cm}^{-1}$ using a $\mathrm{KBr}$ pellet method.

\section{Phytochemical test of extract Test for flavonoids}

$5 \mathrm{~mL}$ of each extract was hydrolyzed with $10 \% \mathrm{v} /$ $\mathrm{v}$ sulfuric acid and cooled. Then, it was extracted with diethyl ether and divided into three portions into three separate test tubes. $1 \mathrm{~mL}$ of diluted sodium carbonate, $1 \mathrm{~mL}$ of $0.1 \mathrm{~N}$ sodium hydroxide, and $1 \mathrm{~mL}$ of strong ammonia solution were added to the first, second and third test tubes, respectively. In each test tube, the development of yellow color demonstrated the presence of flavonoids.

Shinoda's test: Small quantities of extracts were dissolved in alcohol, followed by the addition of one piece of magnesium and concentrated hydrochloric acid $(\mathrm{HCl})$ which were added dropwise and heated. The appearance of the magenta color exhibited the presence of flavonoids.

\section{Test for carbohydrates}

$100 \mathrm{mg}$ of the extract was dissolved in $5 \mathrm{~mL}$ of distilled water and filtered. The filtrate was subjected to the following tests.

Molisch's test: To the $2 \mathrm{~mL}$ of filtrate, two drops of the alcoholic solution of $\alpha$-naphthol were added. The mixture was shaken well, and $1 \mathrm{~mL}$ of concentrated sulfuric acid $\left(\mathrm{H}_{2} \mathrm{SO}_{4}\right)$ was added slowly along the sides of the test tube. The test tube was cooled in ice water and allowed to stand for a few minutes. A violet ring at the junction indicated the presence of carbohydrates.

Fehling's test: $1 \mathrm{~mL}$ of filtrate was boiled in a water bath with $1 \mathrm{~mL}$ each of Fehling's solution A and B. Formation of a red precipitate indicated the presence of sugars.

\section{Test for alkaloids}

About $50 \mathrm{mg}$ of solvent-free extract was stirred with little quantity of dilute hydrochloric acid and filtered. The filtrate was tested carefully with various alkaloid reagents as follows.

Dragendroff's test: To a few $\mathrm{mL}$ of filtrate, 1 or 2 $\mathrm{mL}$ of Dragendorff's reagent was added. A prominent reddish brown precipitate indicated a positive test and hence the presence of alkaloids.

Mayer's test: To a few mL of filtrate, two drops of Mayer's reagent were added along the sides of the test tube. Formation of white or creamy precipitate 
confirmed the test as positive.

Hager's test: To a few $\mathrm{mL}$ of filtrate, 1 or $2 \mathrm{~mL}$ of Hager's reagent was added. A prominent yellow precipitate indicated a positive test.

\section{Test for phenols}

Small quantities of extracts were treated with a neutral ferric chloride solution. The appearance of violet color was taken as a positive test for the presence of phenols.

\section{Test for tannins}

a. Small quantities of extracts were treated with $10 \%$ lead acetate solution. The appearance of white precipitate indicated the presence of tannins.

b. Small quantities of extracts were treated with an aqueous bromine solution. The white precipitate formation was taken as a positive test for the presence of tannins.

\section{Amino acids and proteins}

To $1 \mathrm{~mL}$ extract, 2 drops of freshly prepared $0.2 \%$ ninhydrin reagent were added and heated. The blue color developed indicated the presence of proteins.

\section{Terpenoids}

A volume of $5 \mathrm{~mL}$ of the plant extract was mixed in $2 \mathrm{~mL}$ of chloroform, and concentrated $\mathrm{H}_{2} \mathrm{SO}_{4}$ was added to form a layer. A reddish brown color was formed at the interface, which indicated the presence of terpenoids.

Table 1 Phytochemical tests of extract

\begin{tabular}{|c|c|c|c|}
\hline Sr. No. & Chemical constituent & Chemical test & Significance* \\
\hline \multirow{3}{*}{1} & \multirow{3}{*}{ Alkaloids } & Dragendorff's test & + \\
\hline & & Hagers test & + \\
\hline & & Mayer's test & - \\
\hline 2 & Terpenoids & Salkowski test & + \\
\hline 3 & Phenols & Ferric chloride & + \\
\hline 4 & Flavanoids & Shinoda's & + \\
\hline \multirow{2}{*}{5} & \multirow{2}{*}{ Carbohydrates } & Molisch's test & - \\
\hline & & Fehling's test & + \\
\hline 6 & Amino acids & Ninhydrin & - \\
\hline \multirow{3}{*}{7} & \multirow{3}{*}{ Tannins } & Ferric chloride & + \\
\hline & & & \\
\hline & & Goldbeaters skin test & + \\
\hline
\end{tabular}

* Test results indicating (+) present and (-) absent

\section{Results and Discussion Phytochemical test of extract}

As discussed previously, the prepared extract was examined for the presence of a variety of chemical constituents. The phytochemical screening confirmed the presence of the secondary metabolites, i.e. alkaloids, phenols, glycosides, terpenoids, flavonoids, tannins, etc. [24, 25].

\section{Effect of $\mathrm{pH}$ on the particle size of Ag NPs}

The $\mathrm{pH}$ of the reaction environment or the samples used for dispersion can affect the size, and thus ultimately the stability of the Ag NPs [26]. The particle size of Ag NPs increased with the increase in $\mathrm{pH}$ of the reacting medium, since it would always be around neutral but the addition of some alkalis or a buffer solution might affect the final particle size (Table 2). The medium with $\mathrm{pH}$ value above 8 could be prone to hydrolyzed Ag NPs, which would thus result in immediate generation of Ag NPs with larger particle sizes. For purpose of simplification, we designed our study in four different batches stored for 7 days with similar parameters except $\mathrm{pH}$.

Table 2 Effect of $\mathrm{pH}$ on Ag NPs after 7 days of storage

\begin{tabular}{ccccc}
\hline \multirow{2}{*}{ Sr. No. } & pH & Parent peak & \multicolumn{2}{c}{ After 7 days } \\
\cline { 4 - 5 } & & & Wavelength & Absorbance \\
\hline 1 & 6.5 & 439.5 & 428.42 & 1.194 \\
2 & 7.5 & 439.5 & 443.68 & 0.762 \\
3 & 8.5 & 439.5 & 438.01 & 0.773 \\
4 & 9.5 & 439.5 & 450.5 & 0.476 \\
\hline
\end{tabular}

\section{Mechanism of Ag NPs synthesis}

Various studies predicted the probable mechanisms of Ag NPs synthesis. Strictly speaking, polyphenols in the plant extracts play a major role in the reduction of $\mathrm{Ag}^{+}$ions. Typically, plants enriched in a variety of chemicals such as alkaloids, polyphenol, hydroxyphenol, terpenes, flavonoids, etc, are located mainly in the outer layers of plant tissues. Fortunately, they present in high levels usually in the agro-industrial wastes of the plants, such as shells, outer coverings, husk, etc. Most of the literature investigations reported that phenolic acids were the major bioactive components in the biosynthesis of Ag NPs. Phenolic agents have been reported to possess hydroxyl and carbonyl groups that could inactivate ions through chelation. This chelating ability of phenolic compounds 
is possibly associated with high nucleophilicity of their aromatic rings. Consequently, from the theoretical and practical findings discussed above, we can conclude that Ag NPs were bound to oxygen derived from the hydroxyl groups in compounds based on the formation of a new $\mathrm{C}=\mathrm{O}$ group which might be aldehyde, ketone, or carboxylic acid. Gaining a spare electron from this system results in the acquisition by $\mathrm{Ag}^{+}$ion from $\mathrm{AgNO}_{3}$ solution reduction causes the successful synthesis of Ag NPs. The plausible mechanism involved in the TSE based green synthesis of Ag NPs is represented in Fig. 1(b).
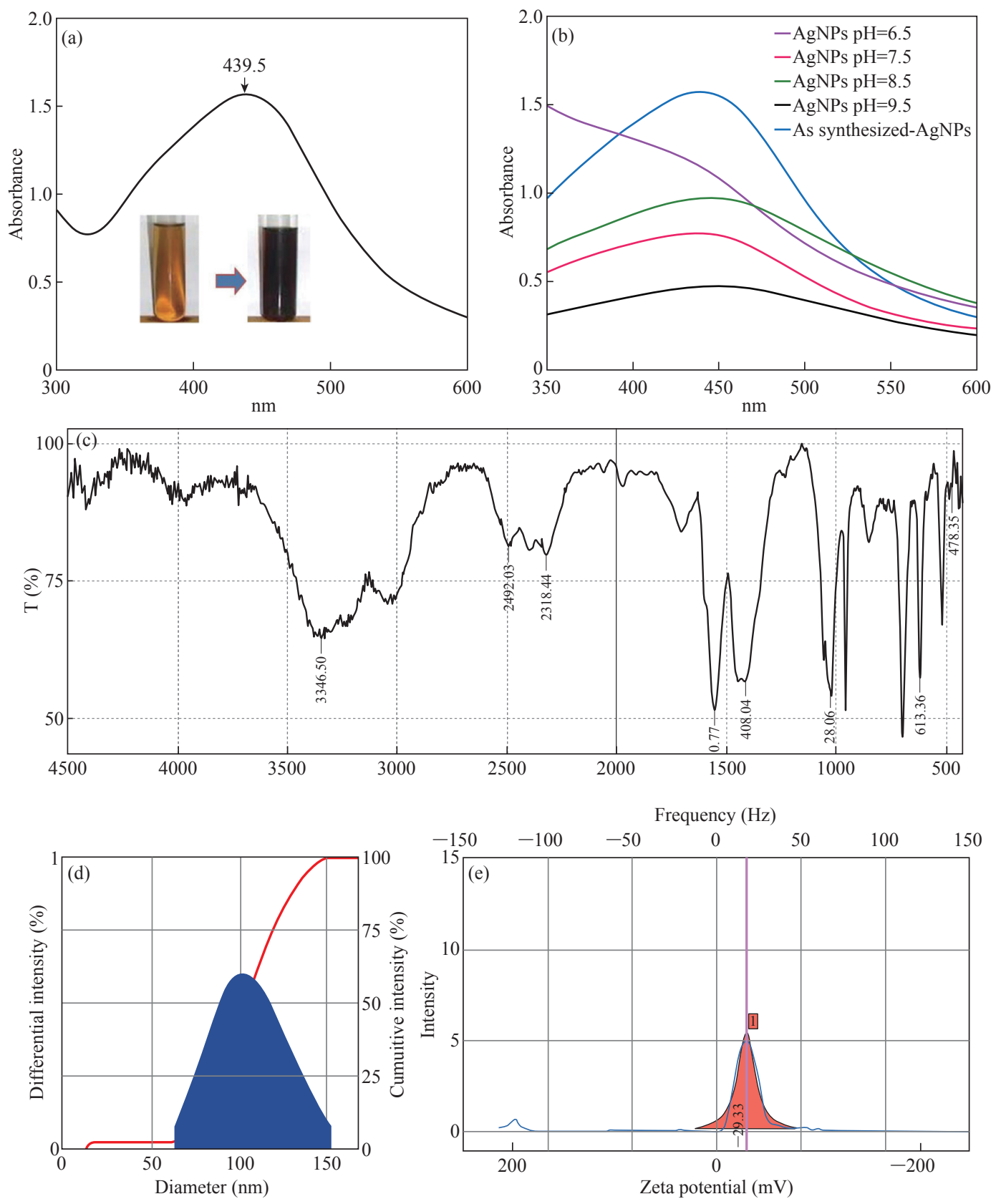

Fig. 2 Ultraviolet-visible spectroscopy of (a) synthesized Ag NPs, (b) after 7-day storage at different pH; (c) Fourier-transform infrared spectroscopy of Ag NPs, (d) particles size and (e) zeta analysis of synthesized Ag NPs.

\section{traviolet-visible spectroscopy (UV-Vis)}

in order to monitor the formation and stability of silver nanoparticles, the absorption spectra of the synthesized silver nanoparticles were recorded against deionized water. Fig. 2(a) shows the ultravioletvisible spectra of silver nanoparticle formation using constant $\mathrm{AgNO}_{3}$ solution (1.0 mM). The typical peak at 439.5 indicated the successful synthesis of Ag NPs. The color of the solutions changed from pale yellow to dark yellowish brown depending on the extract concentration indicating silver nanoparticle formation. Here, the volume of the extract was a variable, which 
Table 3 Effects of $\mathrm{pH}$ on Ag NPs size

\begin{tabular}{ccccccc}
\hline \multirow{2}{*}{ Atches } & $\mathrm{pH}$ & Particle size $(\mathrm{nm})$ & Polydispersity index & \multicolumn{3}{c}{ After 7 days } \\
\cline { 5 - 7 } & & & & Particle size (nm) & Polydispersity index & Zeta potential \\
\hline Batch A & 6.5 & 309.1 & 0.567 & 294.3 & 0.687 & 24.27 \\
Batch B & 7.5 & 290.8 & 0.417 & 293.6 & 0.587 & 9.12 \\
Batch C & 8.5 & 98.1 & 0.362 & 93.7 & 287.5 & -29.33 \\
Batch D & 9.5 & 186.2 & 0.356 & 197.2 & 401.2 & -32.71 \\
\hline
\end{tabular}

could be solely responsible for affecting the results, such as particles size, nature and the yield of Ag NPs. Moreover, to demonstrate the effect of $\mathrm{pH}$, lyophilized powder of Ag NPs was dissolved in solutions of different $\mathrm{pH}$ and incubated at an incubator for 7 days at $28^{\circ} \mathrm{C}$, under normal pressure. The changes in and after 7 days of storage were measured using UV-Vis (Fig. 2(b)). The overall changes due to $\mathrm{pH}$ on the particle size of the Ag NPs are depicted in Table 2.

\section{Fourier-transform infrared spectroscopy (FTIR)}

FTIR was used to investigate the chemical composition of the green synthesized Ag NPs and to identify the probable biomolecules liable for the reduction of the $\mathrm{Ag}^{+}$ions and the capping of the bioreduced silver nanoparticle synthesized by the TSE. The FTIR spectrum of TSE mediated Ag NPs showed bands at $3346 \mathrm{~cm}^{-1}$ which was assigned to the $-\mathrm{NH}$ stretching (Fig. 2(c)). It was reported that the carbonyl groups or amino acid residues in the alkaloidal compounds in TSE were prone to undergo nucleophilic reaction; thus they might act as a capping agent for stabilization of the nanoparticles $[27,28]$. The medium absorption peaks were observed at $3346 \mathrm{~cm}^{-1}$ indicating the N-H stretching, at about $2492 \mathrm{~cm}^{-1}$ due to the $\mathrm{C}-\mathrm{H}$ asymmetric and stretching, and at $1627 \mathrm{~cm}^{-1}$ referring to $\mathrm{C}=\mathrm{O}$. Another peak at $1408 \mathrm{~cm}^{-1}$ showed $\mathrm{C}-\mathrm{C}$ stretching (aromatic), C-H bending at $1477 \mathrm{~cm}^{-1}$, and $1028 \mathrm{~cm}^{-1}$ might be due to the $\mathrm{C}-\mathrm{N}$ stretching. Further, the various functional groups mentioned here might be present due to the variety of compounds present in the TSE extract [29].

\section{Particle size and zeta potential analyses}

The particle size of silver nanoparticles was analyzed on particle size analyzer system (NanoPlus Micromeritics). The average distribution of nanocomposites was based on intensity, volume, and number. The average particle size of the optimized batch was found to be $98.1 \mathrm{~nm}$ and zeta potential value about $29.33 \mathrm{mV}$ (Fig. 2(d) and (e)). The first batch, A operated at slight acidic condition with increased particle size of $309.1 \mathrm{~nm}$ with polydispersity index (PDI) of 0.567 , which might be due to agglomeration in Ag NPs. It was observed that at $\mathrm{pH} 7.5$ the growth of Ag NPs nucleation was easier to occur with the uniform size of about $290.8 \mathrm{~nm}$, with PDI value of 0.417. In the case of batch $C$, it was $98.1 \mathrm{~nm}$ with PDI value of 0.362 . This sudden change in size could be resulted due to an increase in $\mathrm{pH}$. If we went further at increasing $\mathrm{pH}$ at 9.5, there a two-fold increase in size was observed without significant change in PDI [30, 31]. The overall effect of $\mathrm{pH}$ on particles size, zeta potential and PDI are depicted in Table 3.

\section{Scanning electron microscopy-energy dispersive X-ray (SEM/EDX) analysis}

The SEM micrograph of optimized batch showed clustered nature with separated particles (Fig. 3(a)). The particles appeared spherical and a few were rounded cuboidal in shape. Magnified SEM images confirmed that the Ag NPs were in well-resolved structure with soft surface and with sharp edges. It is known that the shape of metal nanoparticle significantly changes its optical and elemental properties [32]. The synthesis of silver nanoparticles from Tamarind shell husk was further characterized by EDX analysis which gave additional evidence for elemental composition of silver nanoparticles. The optical absorption peak was seen approximately at $3 \mathrm{keV}$, which was typical for the absorption of metallic silver nanocrystals due to surface plasma resonance. Moreover, the percent composition of silver was found to be 79.54 and 18.78 of oxygen [33]. The natural precursor may contain some of the chloride traces which is apparent in the graph as of about $1.68 \%$ (Fig. 3(e)). 

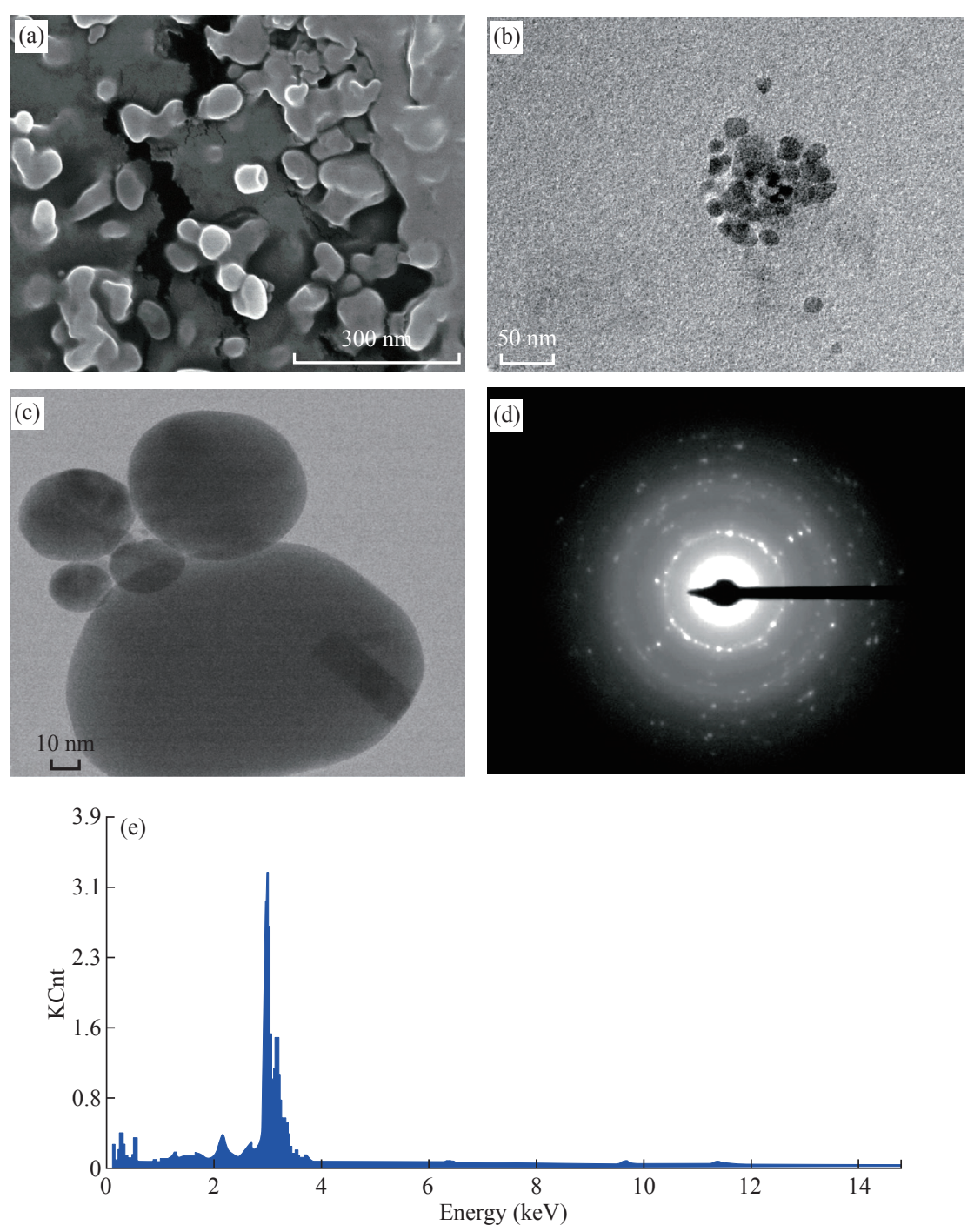

Fig. 3 (a) Scanning electron microscope micrograph, (b) and (c) transmission electron microscopy micrograph, (d) selected area diffraction pattern, and (e) energy dispersive spectroscopy of the Ag NPs.

\section{High resolution transmission electron microscopy (HR-TEM) analysis}

The TSE based synthesized Ag NPs were further characterized by morphological and size analyses using TEM (Fig.3(b) and (c)). We highlighted the TEM results for the optimized batch only. The TEM images captured at different magnifications revealed that some clumps formed measured about $5 \mathrm{~nm}$, and at the side region well dispersed Ag NPs were observed scaling about 5-8 $\mathrm{nm}$ in size. TEM image revealed that the spherical nanoparticles were uniformly distributed without agglomeration. Thus, TEM characterization studies confirmed that the synthesized silver nanoparticles were in nanometer size assortment. The selected area electron diffraction (SAED) pattern of synthesized Ag NPs gave the idea about the even distribution of the particles (Fig. 3(d)). The ring formation at the middle level depicted its crystalline nature [34].

\section{Antimicrobial activity}

The antibacterial activity of different solutions containing Ag NPs demonstrated that two grampositive and a gram-negative bacteria were inhibited by different solutions with different extents. The results of the antibacterial assay are shown in Fig. 4. The gram-negative bacteria $E$. coli was less sensitive to Ag NPs compared with $S$. aureus. This was due to the characteristics of certain bacterial species. We prepared two different concentrations of the synthesized Ag NPs with $2 \mathrm{mM}$ and $4 \mathrm{mM}$ concentrations evaluated for the antimicrobial activity for the different bacterial species respectively. Gentamycin was used as a comparative 


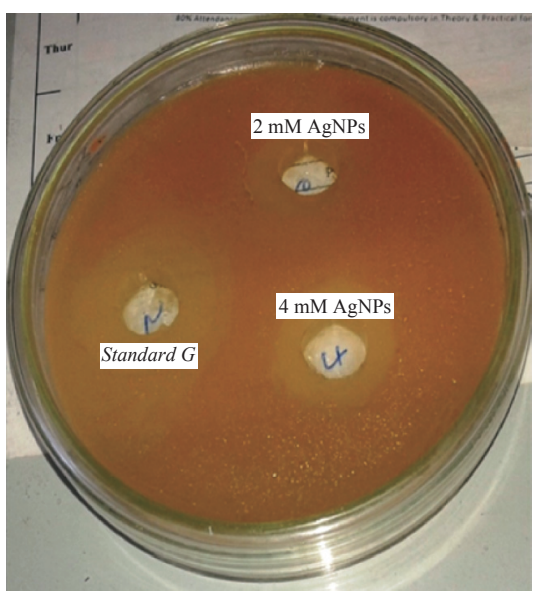

(a) Staphylococcus aureus

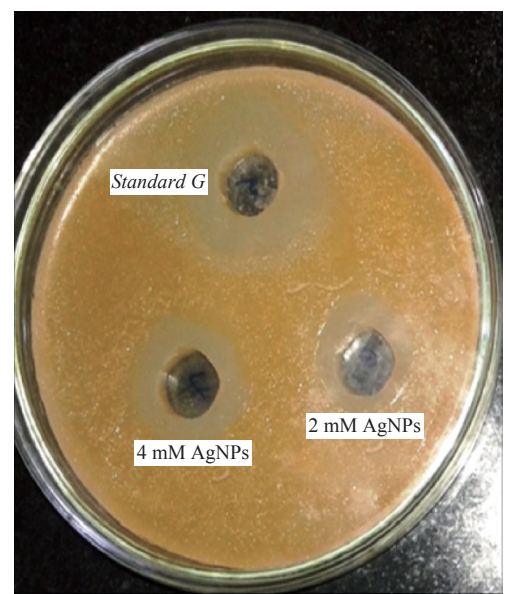

(b) Streptococcus aureus

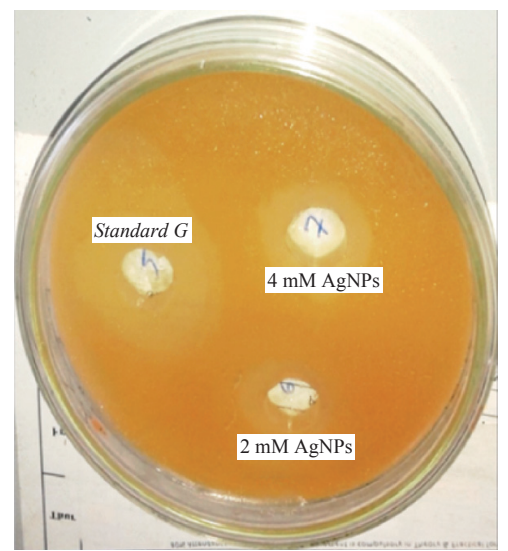

(c) E. coli

Fig. 4 Agar plates with zone of inhibition to respective concentrations of the AgNPs in comparison with standard.

Table 4 The zone of inhibition of the bacteria

\begin{tabular}{cccc}
\hline \multirow{2}{*}{ Sample } & \multicolumn{3}{c}{ Zone of inhibition in (mm) } \\
\cline { 2 - 4 } & Staphylococcus aureus & Streptococcus aureus & Escherichia coli \\
\hline Standard G (gentamycin) & 6.2 & 6.1 & 7.1 \\
Ag NPs 2 mM & 4.5 & 4.6 & 4.0 \\
Ag NPs 4 mM & 2.4 & 2.8 & 2.1 \\
\hline
\end{tabular}

standard for the gram-positive as well as the grampositive strains. It is clearly depicted in the figures that the two different concentrations of Ag NPs showed the distinctive area of bacterial growth suppression. Beside these, gentamycin (standard G) showed equal inhibition of bacterial growth in all three plates. The probable mechanism by which Ag NPs exhibits its antimicrobial actions is still a part of debate and thorough investigations need to be anticipated. Ag NPs primarily undergo dissolution, resulting into "pits" formations. Secondly, the formation of complex with the cellular components of the bacteria (halides and sulfides) results in blockading further cellular processes [34]. Moreover, concentration-dependent accumulation is also an evidence for their antimicrobial properties [35].

\section{Conclusions}

Bestowed with the natures abundant sources of polyphenols, we attempted the synthesis of the Ag NPs. Food agro-industrial waste based synthesis has promoted scientific fraternity toward green synthesis, implementing an eco-friendly approach. We ascribed the synthesis of Ag NPs to tamarind shell extract as green reducing agent. We also studied the stability of the synthesized Ag NPs. Overall results indicated the uniform-size Ag NPs with good stability at the basic $\mathrm{pH}$. In addition, the antibacterial activity of the synthesized Ag NPs was carried out using three different bacterial strains in comparison with gentamycin as standard. These results evidenced the capability of waste biomaterials and revealed a potential application of Tamarind shell extract as a abundant source of reducing agent and dispersant for the biosynthesis of Ag NPs.

\section{Conflict of Interests}

Authors declare no conflict of interest.

\section{References}

[1] M. Govindarajan, M. Rajeswary, S. Hoti, et al., Facile synthesis of mosquitocidal silver nanoparticles using Mussaenda glabra leaf extract: characterisation and impact on non-target aquatic organisms. Natural Product Research, 2016, 30(21): 2491-2494.

[2] V. Kumar, S.K. Yadav, Plant-mediated synthesis of silver and gold nanoparticles and their applications. Journal of Chemical Technology and Biotechnology, 2009, 84(2): 151-157.

[3] M. Rahimi-Nasrabadi, S.M. Pourmortazavi, S.A.S. Shandiz, et al., Green synthesis of silver nanoparticles using Eucalyptus leucoxylon leaves extract and evaluating the antioxidant activities of extract. Natural Product Research, 2014, 28(22): 1964-1969. 
[4] K. Niraimathi, V. Sudha, R. Lavanya, et al., Biosynthesis of silver nanoparticles using Alternanthera sessilis (Linn.) extract and their antimicrobial, antioxidant activities. Colloids and Surfaces B: Biointerfaces, 2013, 102: 288291.

[5] T. Suman, D. Elumalai, P. Kaleena, et al., GC-MS analysis of bioactive components and synthesis of silver nanoparticle using Ammannia baccifera aerial extract and its larvicidal activity against malaria and filariasis vectors. Industrial Crops and Products, 2013, 47: 239-245.

[6] D. Elumalai, P. Kaleena, K. Ashok, et al., Green synthesis of silver nanoparticle using Achyranthes aspera and its larvicidal activity against three major mosquito vectors. Engineering in Agriculture, Environment and food, 2016, 9(1): 1-8.

[7] J.Y. Cheon, S.J. Kim, Y.H. Rhee, et al., Shapedependent antimicrobial activities of silver nanoparticle. International journal of nanomedicine, 2019, 14: 2773.

[8] A. Vala, S. Shah, Rapid synthesis of silver nanoparticles by a marine-derived fungus Aspergillus niger and their antimicrobial potentials. International Journal of Nanoscience and Nanotechnology, 2012, 8(4): 197-206.

[9] S.S. Shankar, A. Ahmad, M. Sastry, Geranium leaf assisted biosynthesis of silver nanoparticles. Biotechnology Progress, 2003, 19(6): 1627-1631.

[10] A. Bankar, B. Joshi, A.R. Kumar, et al., Banana peel extract mediated synthesis of gold nanoparticles. Colloids and Surfaces B: Biointerfaces, 2010, 80(1): 45-50.

[11] J. Parsons, J. Peralta-Videa, and J. Gardea-Torresdey, Use of plants in biotechnology: synthesis of metal nanoparticles by inactivated plant tissues, plant extracts, and living plants. Developments in Environmental Science, 2007, 5: 463-485.

[12] M.S. Akhtar, J. Panwar, and Y.S. Yun, Biogenic synthesis of metallic nanoparticles by plant extracts. ACS Sustainable Chemistry and Engineering, 2013, 1(6): 591602.

[13] J.-P. Rauha, S. Remes, M. Heinonen, et al., Antimicrobial effects of Finnish plant extracts containing flavonoids and other phenolic compounds. International Journal of Food Microbiology, 2000, 56(1): 3-12.

[14] Y.S. Rao, K.M. Mathew, Tamarind handbook of herbs and spices. Elsevier, 2012: 512-533.

[15] S.S. Bhadoriya, A. Ganeshpurkar, J. Narwaria, et al., Tamarindus indica: Extent of explored potential. Pharmacognosy Reviews, 2011, 5(9): 73.

[16] V. Sivasankar, S. Rajkumar, S. Murugesh, et al., Influence of shaking or stirring dynamic methods in the defluoridation behavior of activated tamarind fruit shell carbon. Chemical Engineering Journal, 2012, 197: 162172.

[17] A. Tripathy, A.M. Raichur, N. Chandrasekaran, et al., Process variables in biomimetic synthesis of silver nanoparticles by aqueous extract of Azadirachta indica (Neem) leaves. Journal of Nanoparticle Research, 2010, 12(1): 237-246

[18] Y.Y. Loo, B.W. Chieng, M. Nishibuchi, et al., Synthesis of silver nanoparticles by using tea leaf extract from Camellia sinensis. International Journal of Nanomedicine, 2012, 7: 4263.

[19] G.M. Sulaiman, W.H. Mohammed, T.R. Marzoog, et al., Green synthesis, antimicrobial and cytotoxic effects of silver nanoparticles using Eucalyptus chapmaniana leaves extract. Asian Pacific Journal of Tropical Biomedicine, 2013, 3(1): 58-63.

[20] S.P. Dubey, M. Lahtinen, and M. Sillanpää, Green synthesis and characterizations of silver and gold nanoparticles using leaf extract of Rosa rugosa. Colloids and Surfaces A: Physicochemical and Engineering Aspects, 2010, 364(1-3): 34-41.
[21] S.P. Chandran, M. Chaudhary, R. Pasricha, et al., Synthesis of gold nanotriangles and silver nanoparticles using Aloe vera plant extract. Biotechnology Progress, 2006, 22(2): 577-583.

[22] A. Sharma, S. Kumar, and P. Tripathi, A facile and rapid method for green synthesis of Achyranthes aspera stem extract-mediated silver nano-composites with cidal potential against Aedes aegypti L. Saudi Journal of Biological Sciences, 2019, 26(4): 698-708.

[23] P.S. Nayak, S. Pradhan, M. Arakha, et al., Silver nanoparticles fabricated using medicinal plant extracts show enhanced antimicrobial and selective cytotoxic propensities. IET Nanobiotechnology, 2018, 13(2): 193201.

[24] S. Natukunda, J.H. Muyonga, I.M. Mukisa, Effect of tamarind (Tamarindus indica L.) seed on antioxidant activity, phytocompounds, physicochemical characteristics, and sensory acceptability of enriched cookies and mango juice. Food Science and Nutrition, 2016, 4(4): 494-507.

[25] A. Gomathi, S.X. Rajarathinam, A.M. Sadiq, et al., Phyto chemical screening of aqueous extract of Tamarind (Tamarindus indica L.) Shell. International Journal of Basic and Applied Research, 2017, 7(11): 65-70.

[26] M. Singh, I. Sinha, and R. Mandal, Role of $\mathrm{pH}$ in the green synthesis of silver nanoparticles. Materials Letters, 2009, 63(3-4): 425-427.

[27] P.M. Dewick, Medicinal natural products: a biosynthetic approach. John Wiley and Sons, 2002: 360-380.

[28] D. Balaji, S. Basavaraja, R. Deshpande, et al., Extracellular biosynthesis of functionalized silver nanoparticles by strains of Cladosporium cladosporioides fungus. Colloids and Surfaces B: biointerfaces, 2009, 68(1): 88-92.

[29] T.I. Shalaby, O.A. Mahmoud, G.A. El Batouti, et al., Green synthesis of silver nanoparticles: synthesis, characterization and antibacterial activity. Nanoscience and Nanotechnology, 2015, 5(2): 23-29.

[30] S.K. Balavandy, K. Shameli, D.R. Biak, et al., Stirring time effect of silver nanoparticles prepared in glutathione mediated by green method. Chemistry Central Journal, 2014, 8(1): 11.

[31] M. Yunus, E. Suharyadi, and K. Triyana, Effect of stirring rate on the synthesis silver nanowires using polyvinyl alcohol as a capping agent by polyol process. International Journal on Advanced Science, Engineering and Information Technology, 2016, 6(3): 365-369.

[32] M. Vanaja, K. Paulkumar, G. Gnanajobitha, et al., Herbal plant synthesis of antibacterial silver nanoparticles by Solanum trilobatum and its characterization. International Journal of Metals, 2014

[33] H. Singh, J. Du, P. Singh, et al., Ecofriendly synthesis of silver and gold nanoparticles by Euphrasia officinalis leaf extract and its biomedical applications. Artificial cells, Nanomedicine, and Biotechnology, 2018, 46(6): 11631170.

[34] B. Le Ouay, F. Stellacci, Antibacterial activity of silver nanoparticles: a surface science insight. Nano Today, 2015, 10(3): 339-354.

[35] J.S. Kim, E. Kuk, K.N. Yu, et al., Hwang, Antimicrobial effects of silver nanoparticles. Nanomedicine: Nanotechnology, Biology and Medicine, 2007, 3(1): 95 101

Copyright $($ Rahul Shankar Tade, Sopan Namdev Nangare, and Pravin Onkar Patil. This is an open-access article distributed under the terms of the Creative Commons Attribution License, which permits unrestricted use, distribution, and reproduction in any medium, provided the original author and source are credited. 